\title{
'I can assure you, there is nothing wrong with your kidney'
}

\author{
Author: Tamara Keith ${ }^{\mathrm{A}}$
}

Normal baseline investigation results in a patient with common symptoms is often labelled as being due to a functional disorder, with all the pejorative connotations that go along with that term. When given the opportunity to see a patient for a second opinion, it is important to retain an open mind rather than assuming previous assessments are correct. Such an attitude helps with both attaining the definitive diagnosis but is also crucial to helping give hope to the patient. Understanding the patient's concerns about the meaning of their symptoms is critical in finding the balance between advanced investigation to identify a putative cause versus a decision to proceed with symptomatic control.

KEYWORDS: patient, GP, kidney, history

DOI: 10.7861/clinmed.2020-0990

\section{Case presentation}

A 21-year-old female medical student presented with acute left loin pain, polyuria and a temperature of $37.8^{\circ} \mathrm{C}$. Urinalysis revealed microscopic haematuria, proteinuria and pyuria. Intravenous antibiotics were given for presumed pyelonephritis, however, subsequent urine cultures were negative. Symptoms settled after 5 days. Previous urinalysis over the preceding 2 years had intermittently detected microscopic haematuria, proteinuria and pyuria, which had been treated as urinary tract infections.

Over the following 6 years, there were over 30 hospital admissions with acute throbbing left loin pain, dehydration, polyuria, vomiting and a low-grade fever. Analgesia, intravenous fluids and antibiotics were given until urine cultures returned negative. During these acute episodes, symptoms settled within 72 hours. Urinalysis usually showed microscopic haematuria but never nitrites. Urine culture grew Enterococcus faecalis once, but all other urine cultures were negative. C-reactive protein and white cell count remained normal. Long-term prophylactic antibiotics were prescribed due to the concern that the symptoms were due to recurrent pyelonephritis but made no difference. Between acute episodes, the patient suffered from a constant ache in the left loin, fatigue and polyuria, passing up to five litres of urine in 24 hours, including nocturia.

She was thoroughly investigated with extensive imaging and reviewed by infectious diseases, immunology and second opinion renal physicians. A positive tuberculosis (TB) ELISpot was

Author: ${ }^{\mathrm{A}}$ general practitioner, Cambridgeshire, UK identified, and she was treated for latent TB. No evidence of renal TB was found.

No cause for the recurrent episodes could be found and she was repeatedly told, 'I can assure you, there is nothing wrong with your kidney.'

While on clinical attachment as a medical student, she presented again with an acute episode of left loin pain. Renal ultrasound revealed a 'swollen' left kidney. Magnetic resonance angiography (MRA) followed. The arterial studies were normal, however, the venous images showed compression of the left renal vein between the aorta and the superior mesenteric artery (SMA) consistent with the diagnosis of nutcracker syndrome (Fig 1).

Aspirin was started due to reports of symptomatic benefit but made no significant difference. ${ }^{1}$ Given that weight gain had been reported to provide benefit, the patient, who had a body mass index of $19 \mathrm{~kg} / \mathrm{m}^{2}$, gained $5 \mathrm{~kg}$ in weight which provided only a short-term benefit before a return in symptoms. During this time, severe orthostatic proteinuria was identified. ${ }^{2}$

Definite diagnosis of nutcracker syndrome was confirmed 6 years after presentation, with selective left renal vein venography measuring a renocaval pullback gradient of $7 \mathrm{mmHg}(<1 \mathrm{mmHg}$ in control patients; pressure gradient of $3 \mathrm{mmHg}$ is indicative of renal

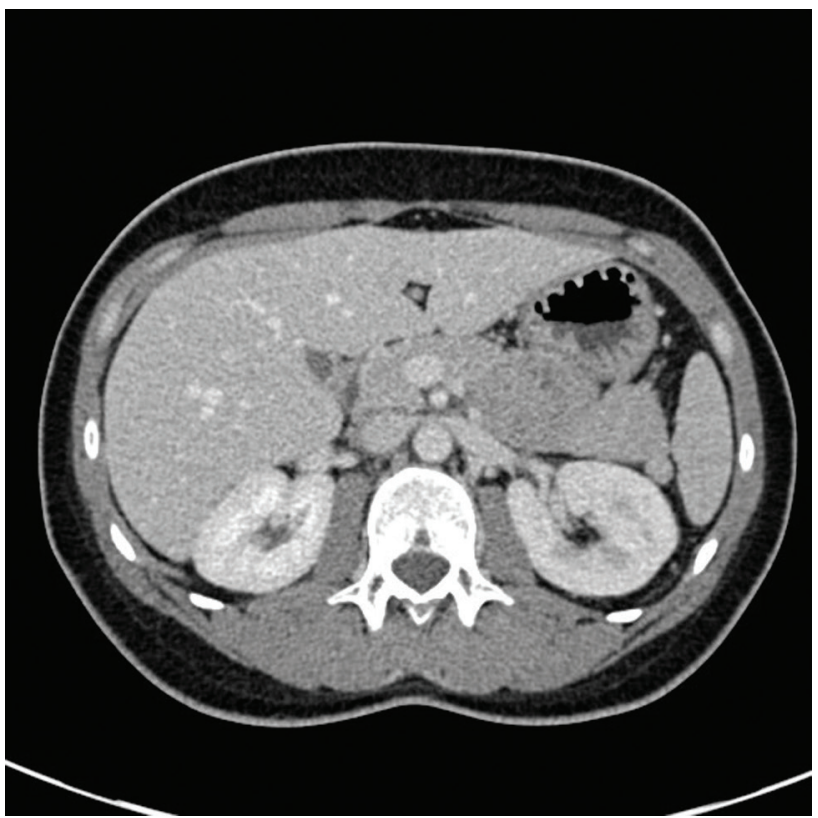

Fig 1. Computed tomography demonstrating left renal vein compression between the superior mesenteric artery and aorta. 
hypertension). ${ }^{3}$ Images taken during venography demonstrated stenosis of the left renal vein caused by the compression between the SMA and aorta with stasis of contrast noted indicating impaired venous outflow accompanied by distension of the distal portion of the left renal vein. It was noted that when contrast was injected into the left renal vein it caused a sudden pain in the left loin, the same as experienced during an exacerbation of nutcracker syndrome.

Treatment options were considered and, due to the debilitating and recurrent nature of her symptoms, surgery was undertaken in the form of left renal autotransplantation. Renal autotransplantation involved a left donor nephrectomy followed by reimplantation of the kidney on the right side. Post-surgery, all symptoms entirely resolved.

Three months postoperatively, the patient had severe headaches with visual symptoms, associated with a blood pressure of 220/118 mmHg. Transplant renal artery stenosis was found (Fig 2). This was stented and normotension returned briefly. This was, unfortunately, ultimately not successful with two episodes of restenosis associated with severe hypertension despite further interventions. Ultimately, a nephrectomy of the autotransplant was performed. She recovered well postoperatively and was not readmitted to hospital.

Seven years have passed since her nephrectomy, she has had no further medical problems and is now a general practice partner and trainer. She is also the author of this article.

\section{Reflections from the wrong side of the stethoscope}

For the whole of my 20s I was a patient, while trying to qualify as a doctor. Although this now feels like a different lifetime, it has shaped the general practitioner (GP) that I am today. Frequently I see patients labelled as 'troublemakers' or their symptoms dismissed as 'functional'. Sadly, so often these labels are applied rapidly in a patient's quest for diagnosis and result in judgemental assumptions, sometimes preventing diagnosis at all.

If 'no cause can be found' that does not mean that there is no cause to be identified and 'but that is incredibly rare' does not mean it is impossible. These phrases were so often used during my

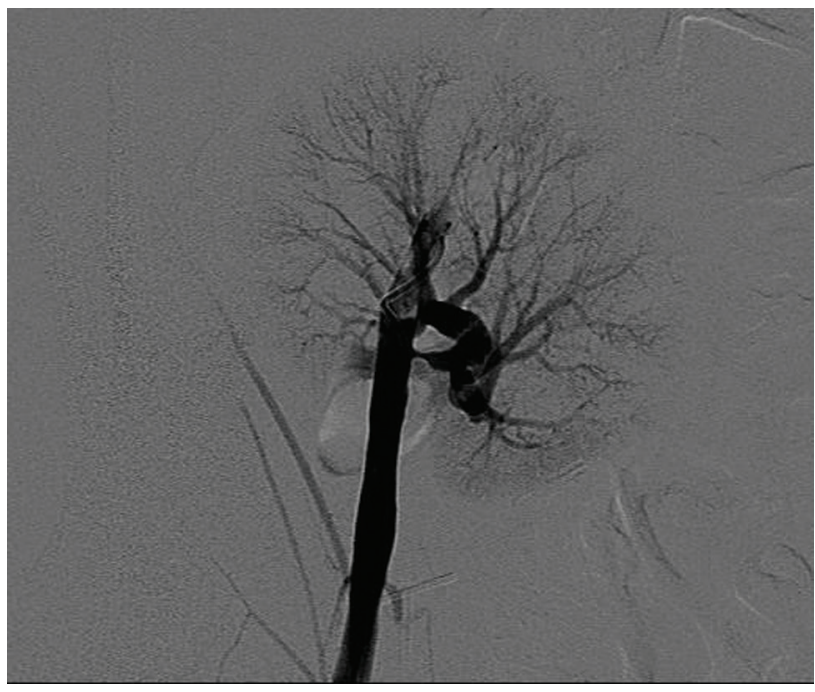

Fig 2. Angiography showing renal artery stenosis. care. When you have a patient in front of you, consider the reason they are consulting and the impact of the symptoms on their life, listening carefully to the patient. Don't prejudge before you have taken a history, avoid jumping to the same conclusion that your team may have, don't label someone without a diagnosis as functional merely because the correct diagnosis has not yet been found. Six years passed from the first presentation where it was clear the pain originated in the left loin in the anatomical position of the kidney to a diagnosis being made.

Upon the finding of the first radiological abnormality (the 'swollen' kidney on ultrasound) the correct diagnostic pathway opened. This radiologist was humble and honest, a refreshing change, simply saying 'I don't know what is wrong, but your left kidney is not normal.' No one wants to be told they have renal disease but when you've been in pain for 6 years with no diagnosis and had multiple admissions which now threaten your entire future, you have no hope. A diagnosis is key to cure, recovery and hope. This was the transformative moment in the diagnostic journey.

Through my own efforts I had arrived at my diagnosis, renal vein compression syndrome (otherwise known as nutcracker syndrome); I then had to prove it.

For 6 years my pain had been discredited, my agenda questioned, my honesty mistrusted by many in the renal team, yet they continued to treat me as suffering from recurrent pyelonephritis. Fortunately, I had a renal physician and radiologist who believed that my symptoms were real and were keen to find the cause. However, there was disagreement within the team thwarting progress. I had been labelled; I was 'functional', the attention seeking medic, the annoying 'expert patient'. This was a real and genuine problem. I had concluded the diagnosis was nutcracker syndrome long before many of them had heard of it, I knew more about this condition than any of them. Consultants do not take kindly to being taught by medical students.

While an MRA identified renal vein compression, venography was not forthcoming. This was disappointing; while not without risks, an increased pressure gradient between the left renal vein and inferior vena cava is considered the gold standard diagnostic test which needs to be undertaken before any surgical intervention. ${ }^{4,5}$

This was one of my lowest moments, being declined a definitive diagnostic procedure which would open the door to curative treatment. Renal vein compression is seen in some asymptomatic individuals and this led to many within the broader renal team to discredit the nutcracker diagnosis. What was notable, however, was those who had labelled me knew very little about me, had mostly not met nor examined me. They were consultants, distant from the patient, simply treating numbers. A critical lesson from this case is to meet the patient and listen to the history, don't just hear the story; listen.

A turning point was when orthostatic proteinuria was found, abnormal numbers now supported the symptoms and radiology. Finally, venography went ahead and confirmed the diagnosis but again a barrier appeared: 'Surgery will open a can of worms.' Indeed, it might have done, but is it not the patient's choice to make an informed decision or was I destined for a life of pain? My renal physician feared breaking the golden rule 'do no harm', fearing surgical complications. In contrast, I argued that doing nothing was far more harmful than proceeding; surgical referral followed. I personally sought treatment opinions in both France and the USA, both supporting my desire for surgery. When it became clear I was considering renal vein transposition in France, I was referred 
to a renal transplant surgeon within the renal centre I was under as well as a second opinion from another renal unit in London. This was now 8 years after the initial presentation.

The opinion from the second renal physician proposed altruistic donation as nephrectomy would cure the condition. My treating team considered this solution both 'dramatic' and risky, while I did not feel there was anything altruistic about donating a frankly dodgy kidney. I was also concerned that if the proteinuria arose from my normal kidney, that a nephrectomy may risk renal failure in later years.

The transplant surgeon listened, understood the problem and offered to proceed with surgery. His proposal was renal autotransplantation, a controversial view within the renal centre.

Autotransplantation had not been done for this condition in the UK before and was major surgery. A condition or treatment may be rare, but this does not mean it is impossible or cannot, or should not, be done.

From the moment I woke after surgery, the nutcracker symptoms had entirely resolved. My sense of relief was palpable; my future was back on track after a decade of uncertainty.

This journey had changed me as a doctor and as a human being. I weigh up all major decisions with the same method I used when considering surgery. What was the worst possible outcome? Losing my kidney if I proceeded or staying in an endless cycle of pain if I did not. I chose the best of these two options; I have no regrets with the decision for autotransplantation. Hindsight is useful, I would be in the same position now had I opted for nephrectomy at the outset but the story could have had numerous worse outcomes.

The challenge in cases like mine is the balance between continued investigation to find a cause versus a decision to proceed with symptomatic control. One of my immense frustrations was that my symptoms were not controlled. Daily diclofenac in a patient with possible renal disease seemed absurd, covering up the pain to make the clinician feel better is not for the benefit of the patient.

When consulting with patients with unexplained symptoms, be it palpitations, abdominal pain or headaches, of course, initially exclude any red flag features. Then exclude any underlying serious cause of pathology before focusing on the impact of the symptoms. Many patients just want reassurance that their symptoms are not serious when the symptom itself is not that bothersome while for others the symptom itself limits activity, saps all energy, ruins relationships and destroys their life - often with chronic pain. As a clinician, the most important part of the history, for me, is the impact the symptom is having on the patient's life; once red flags are excluded, this impact should guide the decision of investigation versus symptomatic control. If a patient becomes stuck in a cycle of investigation with no progress, a second opinion with a fresh set of eyes on the patient can prove fruitful and sometimes a pause in investigation may allow other symptoms and signs to settle or new signs to surface, using time as a diagnostic tool.

I do not hold any individual to 'blame', nor do I see my case as a failure. Significant opportunities to accelerate diagnosis and treatment were missed not because they were ignored but there seemed a fear of causing harm from further investigation and surgical treatment. Ultimately, the team assumed these symptoms were functional and had no appreciation or empathy of the significant impact they had on me. This may have been overcome if the renal team had researched nutcracker syndrome earlier as, with knowledge, missed opportunities were identified and treatment was able to proceed. Moreover, there seemed a lack of empathy as to how disruptive and destructive this illness had become. Each admission was under a different team, the lack of continuity meant no one pieced the story together and because admissions were so frequent, they were normalised. My new normal was not okay!

In hindsight, a second opinion much earlier from a different hospital would have been useful and may have accelerated the diagnosis. Never be afraid as a doctor to say that you do not know what is wrong and ask others for help; humility and honesty are at times more important than knowledge. The most helpful doctor in this story was the one who said he did not know what was wrong but that the left kidney was not right. Reflecting back on the case, it is the diagnosis of recurrent pyelonephritis which should have been questioned early on when all urine cultures apart from one were clear. This highlights the risk when an initial diagnosis is incorrect but follows the patient and is hard to erase.

I have had three memorable patients where I witnessed their struggle similar to my own. No diagnosis had been found and they had been labelled as functional, annoying or mentally ill. They were despairing and had lost all hope. Two of them were tearful young women, who are so often labelled as functional. Both presented with pelvic pain and had had normal investigations. One went on to be diagnosed with endometriosis and the other with ovarian cancer. One elderly man who presented with recurrent hiccups went on to be diagnosed with metastatic renal cancer. With each of them, the critical step was retaking the history from the beginning, repeating the examination and completing relevant investigations. Sometimes the problem has been going on so long the patient doesn't know where to start. The question I always ask is, 'When did you last feel completely well?'

Now I am in a position where I teach medical students and GP trainees, I encourage them to retake a history, question the diagnosis and any prior assumptions made. Start from the beginning and, most of all, listen to the patient. This will take time; time we rarely have but, so often if we do it, saves much more time in return and can potentially save lives.

As a GP, I have the privilege to look after the whole individual, following a patient journey. At times, secondary care clinicians can become stuck in their diagnostic box, responding with: 'It's not my department.' The patient is then passed around various departments or back to the GP and labelled as functional. This can be summarised by the attitude: 'I cannot find what is wrong so there must be nothing wrong.'

In primary care, although we can refer a patient, ultimately, they will boomerang back to us if a cause is not found for their symptoms. Every patient who walks through the door has unexplained symptoms until our history is taken, whereas in secondary care, often the initial differential diagnosis has been made. I worry at times when a patient presents with a 'label' already in place that it can be hard to erase; in my case, each admission was labelled as pyelonephritis following the first presentation.

\section{Conclusion}

Nutcracker syndrome continues to be controversial; it is rare and there is limited research and experience of this condition. There is no agreement on the best treatment approach or even the best means of diagnosis. When conservative management fails and debilitating symptoms remain, surgical options include open or laparoscopic surgery or endovascular stenting to relieve the renal vein compression. There is no single diagnostic criterion, although 
imaging is needed either with ultrasound or cross-sectional imaging to measure the angle of the superior mesenteric artery and look for associated left renal vein compression. Formal venography remains the diagnostic gold standard. ${ }^{6,7}$

Throughout my journey, certain doctors had been judgemental and thoughtless. A diagnosis of nutcracker syndrome had been dismissed due to its rarity and from a lack of knowledge. I had been labelled as functional, possibly in part because I was a medic myself. Physicians followed the herd of disbelief, discrediting the patient and treating numbers alone. Failure to hear and failure to listen.

Open your ears, open your minds, start from the beginning, the answer will be in front of you. 'I can assure you, there is nothing wrong with your kidney.' Yes, they were correct - but the problem was one inch to the right.

\section{Key points}

> 'When did you last feel well?' Take the history from the beginning, without prejudgement or prejudice.

$>$ Work to understand the patient as a whole person - what is the impact of the symptoms on the patient's life. This is key when evaluating the option of further investigation versus supporting the patient to live with their symptoms.

$>$ Rare does not mean impossible.

> Do no harm but work with the patient to evaluate which harm is greater - doing nothing or proceeding with a risky procedure. Remember, with informed consent, high-risk procedures are entirely justified.
$>$ Never be afraid as a doctor to say that you don't know what is wrong; humility and honesty are, at times, more important than knowledge.

\section{References}

1 Shin JI, Lee JS. Can chronic fatigue symptoms associated with nutcracker phenomenon be treated with aspirin? Med Hypotheses 2007:69:704-5.

2 Wendel RG, Crawford ED, Hehman KN. The 'nutcracker' phenomenon: an unusual cause for renal varicosities with hematuria. J Urol 1980;123:761-3.

3 Beinart C, Sniderman KW, Tamura S, Vaughan Jr ED, Sos TA. Left renal vein to inferior vena cava pressure relationship in humans. J Urol 1982;127:1070-1.

4 Rudloff U, Holmes RJ, Prem JT et al. Mesoaortic compression of the left renal vein (nutcracker syndrome): case reports and review of the literature. Ann Vasc Surg 2006;20:120-9.

5 Wang L, Yi L, Yang L et al. Diagnosis and surgical treatment of nutcracker syndrome: a single-center experience. Urology 2009;73:871-6.

6 Ananthan K, Onida S, Davies AH. Nutcracker syndrome: an update on current diagnostic criteria and management guidelines. Eur ] Vasc Endovasc Surg 2017;53:886e894.

7 Macedo GL, Santos MA, Sarris AB, Gomes RZ. Diagnosis and treatment of the Nutcracker syndrome: a review of the last 10 years. J Vasc Bras 2018;17:220-8.

Email: tamara.keith@nhs.net and gives encouragement to trainees across the UK

Winning trainees will be able to present and discuss their lecture in a safe and friendly setting with a diverse audience of peers and senior colleagues. They will receive feedback on their work and presentation, including insights on how to improve.
Don't miss the entry deadline for your region or nation:

21 February - Eastern, KSS, Mersey, Oxford \& Thames Valley, South West and Yorkshire

6 June -- London, Northern Ireland, Northern, North Western, Wessex, West Midlands and Wales 